\title{
Natural selection to sports, later physical activity habits, and coronary heart disease
}

\author{
Urho M Kujala, Seppo Sarna, Jaakko Kaprio, Heikki O Tikkanen, Markku Koskenvuo
}

\begin{abstract}
Objectives-To investigate the associations between natural selection to sports at a young age, continuity of physical activity, and occurrence of coronary heart disease.

Design-Prospective cohort study.

Setting-Finland.

Participants-Former top level male athletes participating at a young age (19201965 ) in different types of sport (endurance $(n=166)$, power speed $(n=235)$, "other" $(n=834))$ and controls healthy at the age of 20 years $(n=743)$.

Main outcome measures-Data on the occurrence of coronary heart disease were obtained from death certificates, three nationwide registers, and questionnaire studies in 1985 and 1995, and data on later physical activity were obtained from the questionnaires.

Results-In 1985 all groups of former athletes were more physically active than controls $(p<0.001)$. Despite similar total volumes of physical activity, compared with power speed athletes, former endurance athletes participated more often in vigorous activity $(p=0.006)$ and had less coronary heart disease (adjusted odds ratio $0.34,95 \%$ confidence interval 0.17 to $0.73 ; p=0.004)$. In 1985 and 1995 , both endurance and other athletes had less coronary heart disease than controls. From 1986 to 1995, the incidence of new coronary heart disease was lower among those who participated in vigorous physical activity in 1985.

Conclusions-Both a previous aptitude for endurance athletic events and continuity of vigorous physical activity seem to be associated with protection against coronary heart disease, but an aptitude for power speed events does not give protection against coronary heart disease.

(Br f Sports Med 2000;34:445-449)
\end{abstract}

Keywords: coronary heart disease; fitness; genetic selection; physical activity

High amounts of leisure physical activity correlate with physical fitness, ${ }^{1}$ but physical fitness also has a genetic component. ${ }^{2}$ High levels of both physical fitness and activity, ${ }^{3-7}$ and in particular high levels of lifelong vigorous endurance training, ${ }^{8}$ contribute to a low risk of coronary heart disease. However, genetic variability seems to make it easier for some people to achieve a physically active lifestyle and high levels of physical fitness, as well as favour them with reduced occurrence of coronary heart disease. ${ }^{89}$ Inherited differences in the properties of skeletal muscle may be one such selective mechanism.

Genetic factors contribute most to the high interindividual variability in the proportion of slow twitch (type I) muscle fibres with high oxidative capacity, ${ }^{10}$ which correlate positively with both physical fitness and a favourable lipid profile (high levels of high density lipoproteincholesterol and low levels of triglyceride). ${ }^{11}{ }^{12}$ Compared with endurance athletes, who have a high percentage of slow twitch muscle fibres, power athletes and sprinters have a high proportion of fast twitch (type II) muscle fibres with low oxidative capacity. ${ }^{13}$ In addition to an unfavourable lipid profile, a high proportion of fast twitch fibres has been associated with obesity, ${ }^{14}$ hypertension, ${ }^{15}$ and insulin resistance. ${ }^{16}$ Accordingly, men with natural ability in power sports seem to have a higher risk of developing cardiovascular disorders than those with natural ability in endurance sports. ${ }^{8} 17$

The aim of this study was to estimate the impact of genetic selection compared with physical activity in later years as predictors of coronary heart disease by studying former endurance athletes, power speed athletes, other athletes, and population controls who were healthy at the age of 20 .

\section{Methods}

We investigated continuity of physical activity and occurrence of coronary heart disease among former athletes and controls by questionnaire studies and consulting three nationwide registers and death certificates.

SUBJECTS

Male athletes who had represented Finland between 1920 and 1965 at least once at the Olympic games, World or European championships, or other international competitions in selected sports were identified. ${ }^{18}$ The full name, place, and date of birth were traced for $98 \%$ of the athletes from selected sports. Controls were selected from among Finnish men who at about 20 years of age had been classified as completely healthy (military class AI, fully fit for ordinary military service) at the medical examination preceding their conscription. ${ }^{18}$ They were drawn from the public archives of the register of men liable for military service and matched for birth cohort and area of residence with the athletes. The original cohort of athletes included 2401 men and the reference group 1712 men. ${ }^{18}$

In 1985,1282 (response rate $80-90 \%$ by sport of those alive in 1985) athletes and 777 (response rate $77 \%$ ) controls responded to our 
Table 1 Characteristics of the final study cohort (subjects responding to the 1985 questionnaire with complete data)

\begin{tabular}{|c|c|c|c|c|}
\hline & $\begin{array}{l}\text { Endurance athletes } \\
(n=166)\end{array}$ & $\begin{array}{l}\text { Power speed } \\
\text { athletes }(n=235)\end{array}$ & $\begin{array}{l}\text { Other athletes } \\
(n=834)\end{array}$ & $\begin{array}{l}\text { Controls } \\
(n=743)\end{array}$ \\
\hline Mean (SD) age in 1985 (range) (years) & $\begin{array}{l}61.6(11.0) \\
(43.2-89.7)\end{array}$ & $\begin{array}{l}56.9(10.9) \\
(38.6-88.4)\end{array}$ & $\begin{array}{l}55.4(10.5) \\
(35.9-93.7)\end{array}$ & $\begin{array}{l}54.8(10.1) \\
(38.1-86.4)\end{array}$ \\
\hline Mean (SD) body mass index in 1985 (range) $\left(\mathrm{kg} / \mathrm{m}^{2}\right)$ & $\begin{array}{l}24.4(2.5) \\
(19.2-32.5)\end{array}$ & $\begin{array}{l}27.1(3.5) \\
(19.7-41.3)\end{array}$ & $\begin{array}{l}26.1(3.3) \\
(16.2-43.3)\end{array}$ & $\begin{array}{l}26.4(3.6) \\
(15.8-58.1)\end{array}$ \\
\hline \multicolumn{5}{|l|}{ Smoking status (n (\%)) } \\
\hline Non-smokers & $110(66.3)$ & $121(51.5)$ & $417(50.0)$ & $211(28.4)$ \\
\hline Ex-smokers & $39(23.5)$ & $84(35.7)$ & $278(33.3)$ & $345(46.4)$ \\
\hline Current smokers & $17(10.2)$ & $30(12.8)$ & $139(16.7)$ & $187(25.2)$ \\
\hline \multicolumn{5}{|l|}{ Occupational group (n (\%)) } \\
\hline Executives & $9(5.4)$ & $67(28.5)$ & $233(27.9)$ & $90(12.1)$ \\
\hline Clerical staff & $84(50.6)$ & $96(40.9)$ & $330(39.6)$ & $197(26.5)$ \\
\hline Skilled workers & $46(27.7)$ & $60(25.5)$ & $236(28.3)$ & $311(41.9)$ \\
\hline Unskilled workers & $7(4.2)$ & $7(3.0)$ & $12(1.4)$ & $35(4.7)$ \\
\hline Farmers & $20(12.1)$ & $5(2.1)$ & $23(2.8)$ & $110(14.8)$ \\
\hline
\end{tabular}

questionnaire. ${ }^{8}$ For the present study we selected only athletes and controls who responded with a complete set of data to the 1985 questionnaire (table 1). From the athletes, we selected two extreme groups according to the presumed ${ }^{19}$ muscle fibre composition suspected of giving the best advantage for a specific type of sports event and thus being a selective factor for natural selection to different types of sport. We assigned endurance runners $(n=101)$ and cross country skiers $(n=65)$ to the "endurance" athlete group, and sprinters $(n=73)$, weightlifters $(n=66)$, and track and field throwers (22 shot putters, 24 discus throwers, 20 hammer throwers, 30 javelin throwers) to the "power speed" athlete group. The third athletic group was "other" comprising 154 soccer, 108 ice hockey, and 67 basketball players, as well as 131 boxers, 131 wrestlers, and 243 men from other than the aforementioned track and field disciplines.

DATA SOURCES

Data were collected from the 1985 and 1995 questionnaires, and morbidity data were compiled from three nationwide registers covering all citizens in Finland: the registers for hospital inpatient discharges, reimbursable medications, and disability pensions. ${ }^{8}$ In addition, causes of death were available from the cause of death bureau files at the Central Statistical Office of Finland. The personal identification (social security) code assigned to all Finnish citizens permitted accurate computerised record linkage. Based on registers and death certificates the occurrence of coronary heart disease was analysed using the International classification of diseases (ICD) codes (ICD-8 from 1970 to 1985 and ICD-9 from 1986 to 1995), ${ }^{20}{ }^{21}$ rubric code $410-414$ indicating coronary heart disease.

Between 1986 and 1995, 232 (18.8\%) of the 1235 athletes and $158(21.3 \%)$ of the 743 controls died. In 1995, 931 (91.9\%) of the 1013 athletes and $535(91.5 \%)$ of the 585 controls alive in 1995 responded to the second questionnaire. For those who died during the last 10 year follow up or did not respond to the 1995 questionnaire, the follow up data were derived from death certificates and morbidity registers.

The 1985 and 1995 questionnaires included items on physical attributes (height, current weight), occupation, smoking, physical activity, and any diseases verified by a doctor. The questionnaire included the question: has a doctor said that you have or have ever had angina pectoris (chest pain due to ischaemic/ coronary heart disease) or myocardial infarction?

Data on cause of death were available from 1936 until 31 December 1995. Since 1967 all hospital discharges in Finland have been recorded annually in a nationwide register at the National Board of Health. This is obligatory for all public and private hospitals. Record linkage was not possible until 1969, because the data were too incomplete for accurate identification. Thus the follow up with hospital records in the present study started from the beginning of 1970 and continued until 31 December 1995 . The validity of the register for epidemiological studies of coronary heart disease and myocardial infarction has been shown to be very good. ${ }^{22}{ }^{23}$ Data on disability pensions and reimbursable medication were obtained from the register of the Social Insurance Institution, the public agency responsible for basic social security covering all residents of Finland. ${ }^{8}$ The follow up of the disability pension records started from 1970, when pensions granted earlier in surviving subjects were coded, and continued until 1985. Detailed data on reimbursements because of coronary heart disease were available from 1986 to 1995 .

DETERMINATION OF CORONARY HEART DISEASE

Subjects who had coronary heart disease, including angina pectoris or myocardial infarction diagnosed by a doctor, based on the 1985 questionnaire or at least one of the registers before 1 January 1986, were determined to have coronary heart disease in 1985. Those with coronary heart disease in 1985 or coronary heart disease based on the 1995 questionnaire, or on the hospital discharge registry, the reimbursable medication registry, or death certificates before 1 January 1996, were determined to have coronary heart disease in 1995. Those who had coronary heart disease in 1995 but not in 1985 were considered to be incident cases of coronary heart disease during the last 10 year follow up.

PHYSICAL ACTIVITY

We divided our subjects into two classes according to their participation in vigorous activity in 1985 . Those whose exercise intensity 
Table 2 Odds ratios for coronary heart disease

\begin{tabular}{|c|c|c|c|c|}
\hline & $\begin{array}{l}\text { Endurance athletes } \\
(n=166)\end{array}$ & $\begin{array}{l}\text { Power speed athletes } \\
(n=235)\end{array}$ & $\begin{array}{l}\text { Other athletes } \\
(n=834)\end{array}$ & $\begin{array}{l}\text { Controls } \\
(n=743)\end{array}$ \\
\hline \multicolumn{5}{|c|}{ Coronary heart disease in 1985} \\
\hline $\mathrm{n}(\%)$ & $13(7.8 \%)$ & $36(15.3)$ & $103(12.4)$ & $138(18.6)$ \\
\hline Odds ratio $(95 \% \mathrm{CI})^{\star}$ & $\begin{array}{l}0.21(0.11-0.39) \\
\mathrm{p}<0.001\end{array}$ & $\begin{array}{l}0.65(0.42-0.99) \\
\mathrm{p}=0.041\end{array}$ & $\begin{array}{l}0.56(0.42-0.75) \\
\mathrm{p}<0.001\end{array}$ & 1.0 \\
\hline Odds ratio $(95 \% \mathrm{CI}) \dagger$ & $\begin{array}{l}0.25(0.13-0.47) \\
\mathrm{p}<0.001\end{array}$ & $\begin{array}{l}0.79(0.51-1.23) \\
\mathrm{p}=0.29\end{array}$ & $\begin{array}{l}0.65(0.48-0.89) \\
p=0.006\end{array}$ & 1.0 \\
\hline Odds ratio $(95 \% \mathrm{CI}) \ddagger$ & $\begin{array}{l}0.33(0.17-0.63) \\
\mathrm{p}<0.001\end{array}$ & $\begin{array}{l}0.79(0.51-1.24) \\
\mathrm{p}=0.30\end{array}$ & $\begin{array}{l}0.71(0.52-0.98) \\
p=0.032\end{array}$ & 1.0 \\
\hline \multicolumn{5}{|c|}{ Coronary heart disease in 1995} \\
\hline $\mathrm{n}(\%)$ & $34(20.5)$ & $68(28.9)$ & $182(21.8)$ & $218(29.3)$ \\
\hline Odds ratio $(95 \% \mathrm{CI})^{\star}$ & $\begin{array}{l}0.38(0.25-0.59) \\
\mathrm{p}<0.001\end{array}$ & $\begin{array}{l}0.84(0.60-1.18) \\
p=0.31\end{array}$ & $\begin{array}{l}0.62(0.49-0.79) \\
\mathrm{p}<0.001\end{array}$ & 1.0 \\
\hline Odds ratio $(95 \% \mathrm{CI}) \dagger$ & $\begin{array}{l}0.45(0.29-0.71) \\
\mathrm{p}<0.001\end{array}$ & $\begin{array}{l}1.02(0.72-1.46) \\
\mathrm{p}=0.90\end{array}$ & $\begin{array}{l}0.73(0.56-0.94) \\
\mathrm{p}=0.013\end{array}$ & 1.0 \\
\hline Odds ratio $(95 \% \mathrm{CI}) \ddagger$ & $\begin{array}{l}0.60(0.38-0.95) \\
p=0.027\end{array}$ & $\begin{array}{l}1.02(0.71-1.46) \\
p=0.93\end{array}$ & $\begin{array}{l}0.80(0.61-1.03) \\
p=0.083\end{array}$ & 1.0 \\
\hline
\end{tabular}

${ }^{\star}$ Odds ratios and $\mathrm{p}$ values compared with controls and adjusted for age.

†Odds ratios and $\mathrm{p}$ values adjusted for age, occupational group, and smoking status.

¥Odds ratios and $\mathrm{p}$ values adjusted for age, occupational group, smoking status, participation in vigorous activity in 1985, physical activity MET index in 1985, and body mass index in 1985.

usually corresponded to jogging or running were vigorous exercisers. ${ }^{24}$

The volume of physical activity in 1985 was computed from responses to three structured questions. $^{24}$ We calculated a leisure activity metabolic equivalent (MET) index (cumulative leisure MET-hours/week) by assigning a multiple of resting metabolic rate (MET score) to activity, and by calculating the product of intensity $\times$ duration $\times$ frequency of activity. ${ }^{24}$

CONFOUNDING FACTORS

Occupational data were collected partly from the Central Population Registry and partly from the 1985 questionnaire study. The main occupational groups were as follows: executives, clerical staff, skilled workers, unskilled workers, and farmers. ${ }^{25}$ Each person was classified according to the occupation that they had held longest.

The calculation of body mass index (weight (kg) divided by height $\left(\mathrm{m}^{2}\right)$ ) in 1985 was based on the self reported height and weight in the questionnaire.

Tobacco smoking status of the subjects was determined from their responses to a detailed smoking history. ${ }^{26}$ Non-smokers were men who had smoked no more than five to ten packs of cigarettes (or equivalent of other tobacco product) throughout their lifetime. Other subjects were classified as current smokers or ex-smokers according to whether they were smoking daily at the time of the questionnaire or had quit (table 1).

\section{STUDY DESIGN AND STATISTICAL ANALYSIS}

We analysed the extent to which selection to specific types of sports at a young age predicted later physical activity (in 1985) and prevalence of coronary heart disease (in 1985 and 1995). Differences in physical activity between groups in 1985 were analysed using analysis of covariance, and the Newman-Keuls test was used for post hoc comparisons. We calculated the odds ratios and their $95 \%$ confidence intervals $(95 \%$ CI) for coronary heart disease in 1985 and 1995 for different athletic groups compared with controls using logistic regression models (BMDP Statistical Software). To test our hypothesis we also compared endurance athletes with power speed athletes. Age, body mass index, smoking status, and occupational group were included as confounding factors in the analysis. Here $\mathrm{p}$ values are from Wald's test.

Next, we analysed those without coronary heart disease in 1985 to determine whether selection to sports at a young age or activity in 1985 was the stronger predictor for coronary heart disease in later life. We calculated the odds ratios and their 95\% CIs for the occurrence of coronary heart disease from 1986 to 1995 using logistic regression models, adding the physical activity variables in 1985 to the aforementioned confounding factors.

\section{Results}

All the athlete groups (endurance $36.8 \%$, power speed $28.1 \%$, and others $38.1 \%$ ) participated more often in vigorous physical activity in 1985 than the controls $(11.8 \%$, age adjusted $\mathrm{p}<0.001$ for all comparisons between athlete groups and controls). The mean (SD) total volume of leisure physical activity in 1985 in terms of the leisure activity MET index was 37.7 (41.9) for endurance, 31.6 (38.9) for power speed, 28.5 (31.9) for other athletes, and 15.0 (23.7) for controls (age adjusted $\mathrm{p}<0.001$ for all comparisons between athlete groups and controls). Former endurance athletes participated more often in vigorous physical activity than former power speed athletes (age adjusted $\mathrm{p}=0.006)$ and had a higher MET index (age adjusted $\mathrm{p}=0.015$ ).

In 1985 and 1995, after adjustment for age, occupational group, and smoking, both endurance and other athletes had less coronary heart disease than controls, whereas the difference between controls and power speed athletes was not significant (table 2). After the same adjustments, and in accordance with our hypothesis, former endurance athletes had significantly less coronary heart disease than power speed athletes in both 1985 (odds ratio 0.34, 95\% CI 0.17 to $0.73 ; \mathrm{p}=0.004$ ) and 1995 (odds ratio $0.51,95 \%$ CI 0.30 to $0.87 ; \mathrm{p}=0.015$ ).

Even though the 1985 group differences in prevalence of coronary heart disease persisted in 1995, there were no group differences in the 
incidence of coronary heart disease from 1986 to 1995 among those without manifest coronary heart disease in $1985(\mathrm{n}=1688)$. In contrast, among those free of coronary heart disease in $1985,15.4 \%(181 / 1174)$ of the nonparticipants in vigorous physical activity had coronary heart disease before 1996, compared with $6.0 \%(31 / 514)$ of those who did participate in vigorous activity (age adjusted $\mathrm{p}<0.001$ ). The mean (SD) MET index in 1985 was 26.5 (24.1) in those who did not acquire coronary heart disease during the next 10 years, compared with 20.4 (34.5) in those who did (age adjusted $\mathrm{p}=0.042$ ). We included all variables (age, occupational group, smoking status, body mass index, study group, participation in vigorous activity, leisure physical activity MET index) in a fixed logistic regression model to discover the most important predictors of occurrence of new cases of coronary heart disease from 1986 to 1995 . In this model, the odds ratio for coronary heart disease increased by $4 \%$ (95\% CI 3\% to $6 \%$; $\mathrm{p}<0.001)$ per year of increasing age, and $7 \%$ $(95 \%$ CI $2 \%$ to $12 \%$; $=0.003)$ per unit of increasing body mass index; the odds ratio was increased for smokers compared with nonsmokers (odds ratio $1.57,95 \%$ CI 1.04 to 2.38; $\mathrm{p}=0.030$ ), and decreased for those who participated in vigorous activity compared with those who did not (odds ratio $0.57,95 \%$ CI 0.34 to $0.94 ; \mathrm{p}=0.025)$.

\section{Discussion}

Identification of specific genes that increase susceptibility to common diseases has heightened our awareness of the importance of genetic factors in morbidity. The relative importance of factors over which a person has little or no control, such as genes, compared with those that can be modified, such as smoking and physical activity, needs further clarification. Our study provides evidence that both genetic selection and continuity of physical activity contribute to low occurrence of coronary heart disease among former endurance athletes.

Invasive examination to diagnose coronary heart disease in this epidemiological follow up study was impossible. However, we consider the coverage and specificity of our documentation of coronary heart disease to be good because we recorded the occurrence of coronary heart disease diagnosed by a doctor from self reports in questionnaires as well as from three registers. Register data alone somewhat underestimate morbidity in the Finnish population; however, the registers of reimbursable medication and disability pension are based on expert reviews and record very accurately cases of chronic coronary heart disease, ${ }^{8}$ while the validity of data from the hospital discharge register is sufficiently good for comparative study. ${ }^{22}{ }^{23}$ Death certificates were also used to document coronary heart disease among those who died during follow up. In addition, we analysed mortality from coronary heart disease before 1985 (data not shown) among our study groups: the lower mortality among former endurance athletes compared with controls until 1985 makes our study design conservative. We also adjusted our results for factors that we had earlier found to be associated with occurrence of coronary heart disease in our cohort. ${ }^{8}$ However, because the smoking habits were very different between the groups, it may be that we were not able to eliminate all covariance.

Based on earlier observational studies, great physical fitness, self selected endurance-type leisure, and work related physical activity are all associated with lower occurrence of future coronary heart disease. ${ }^{3-7}$ Compared with controls, our athletes remained physically more active during old age. It is difficult to say how much of this difference is explained by a genetically determined ability to exercise and how much by environmental factors. Although the total volume of physical activity and participation in vigorous activity in old age was much higher among power speed athletes than controls, the prevalence of coronary heart disease did not differ significantly from that of controls and was significantly higher than among endurance athletes. Here, an influence of genetic factors on the occurrence of coronary heart disease is very probable. It is highly unlikely that short term participation in various types of sport at a young age explains differences in occurrence of coronary heart disease in later years. This may account for the varying results seen in studies of the life expectancy of former athletes from different types of sport. ${ }^{27}$ Corresponding analyses in today's athletes may reflect other than physiological differences because of the current widespread abuse of doping substances.

There may be numerous social, psychological, and biological determinants of the successful athlete and possibly also of the risk of future coronary heart disease. One strong candidate is the properties of skeletal muscle. Human muscle contains a mixture of slow twitch and fast twitch muscle fibres, interindividual variation of muscle fibre types in a specific muscle being $10-90 \%$, with a population mean of $50 \% .{ }^{19}$ Endurance capacity has been related to a predominance of slow twitch fibres $(>50 \%$ ), whereas fast twitch fibres are related to power and speed capacity. ${ }^{19}$ Significant changes in muscle fibre composition have not been observed with aging, ${ }^{28}$ and changes during training have fallen within the sampling error of $15 \% .{ }^{10}$ Muscle fibre composition is also associated with many metabolic and cardiovascular variables and thus modifies the risk of coronary heart disease. ${ }^{11}{ }^{12}{ }^{14-16}$ Thus the clustering of risk factors for coronary heart disease in people with a high proportion of slow twitch fibres, such as our endurance athletes, suggests that muscle fibre distribution may explain some of the selection in our athletes, as well the continuity of physical activity and low occurrence of coronary heart disease. This needs to be confirmed by monitoring subjects whose muscle fibre type has been determined.

Although we compared athletes from different types of sport, our findings on genetic selection should also be considered when interpreting the results of population studies. 
Morris et $a l,{ }^{29}$ who originally reported an association between work related physical activity and low coronary heart disease risk among London busmen, found that, compared with conductors, drivers with a high risk of coronary heart disease had bigger uniforms at entry. ${ }^{9}$ Recently, Lean and $\operatorname{Han}^{17}$ reported in a non-athletic sample that men with natural ability in power sports at a young age seemed to have a higher risk of developing cardiovascular disorders than those with a natural ability in endurance sports.

In conclusion, our study provides evidence that natural selection to sports or physical activity at a young age predicts participation in physical activity in later life, as well as low occurrence of future coronary heart disease. However, participation in vigorous physical activity in later life also predicts low occurrence of coronary heart disease, irrespective of selection to sports at a young age. We suggest that genetic selection and vigorous physical activity both contribute to the association between physical activity and coronary heart disease.

We thank Richard Burton for editing the language. The work was supported by grants from the Finnish Ministry of Education, Juho Vainio Foundation, and Academy of Finland (no 42044). There were no competing interts.

Contriter Contributors: UMK, SS, and JK all contributed to formulation of the study design, obtaining funding, collection of data, analysis and interpretation of the data, and writing of the paper. HOT contributed to formulation of the study design, interpretation of the data, and writing of the paper. MK contributed to obtaining funding, collection of data, interpretation of the data,
and writing of the paper. UMK and SS will act as guarantors.

1 Kujala UM, Viljanen T, Taimela S, et al. Physical activity, $\mathrm{VO}_{2 \max }$, and jumping height in an urban population. Med Sci Sports Exerc 1994;26:889-95.

2 Bouchard C, Dionne FT, Simoneau J-A, et al. Genetics of aerobic and anaerobic performances. Exerc Sports Sci Rev 1992;20:27-58

3 Morris JN, Everitt MG, Pollard R, et al. Vigorous exercise in leisure-time: protection against coronary heart disease. Lancet 1980;ii: $1207-10$

4 Paffenbarger RS, Hyde RT, Wing AL, et al. The association of changes in physical activity level and other lifestyle characteristics with mortality among men. $N$ Engl f Med 1993; 324:538-45.

5 Lakka TA, Venäläinen JM, Rauramaa R, et al. Relation of leisure-time physical activity and cardiorespiratory fitness to the risk of acute myocardial infarctation in men. N Engl F Med 1994;330:1549-54.

6 Blair SN, Kohl HW III, Barlow CE, et al. Changes in physical fitness and all-cause mortality: a prospective study of cal fitness and all-cause mortality: a prospective study
healthy and unhealthy men. $\mathscr{F A M A}$ 1995;273:1093-8.

7 Erikssen G, Liestøl K, Bjørnholt J, et al. Changes in physical fitness and changes in mortality. Lancet 1998;352:759-62.
8 Kujala UM, Kaprio J, Taimela S, et al. Prevalence of diabetes, hypertension, and ischemic heart disease in diabetes, hypertension, and ischemic heart dise

9 Morris JN, Heady JA, Raffle PAB. Physique of London busmen. Epidemiology of uniforms. Lancet 1956;ii:569-70

10 Simoneau JA, Bouchard C. Genetic determinism of fiber type proportion in human skeletal muscle. FASEB 7 1995; 9:1091-5.

11 Tikkanen HO, Härkönen $M$, Näveri $H$. Relationship of skeletal muscle fiber type to serum high density lipoprotein cholesterol and apolipoprotein A-I levels. Atherosclerosis 1991;90:49-57.

12 Tikkanen HO, Hämäläinen E, Sarna S, et al. Association between skeletal muscle properties, physical fitness, physical activity and coronary heart disease risk factors in men. Atherosclerosis 1998;137:337-89.

13 Bergh U, Thorstensson A, Sjodin B, et al. Maximal oxygen uptake and muscle fiber types in trained and untrained humans. Med Sci Sports 1978;10:151-4.

14 Wade AJ, Marbut MM, Round JM. Muscle fiber type and aetiology of obesity. Lancet 1990;335:805-8

15 Juhlin-Dannfelt A, Frisk-Holmberg M, Karlsson J, et al. Central and peripheral circulation relation to muscle fiber. composition in normal and hypertensive men. Clin $\mathrm{Sci}$ 1979;56:335-40.

16 Lillioja S, Young AA, Cutler CL, et al. Skeletal muscle capillary density and fibre type are possible determinants of in vivo insulin resistance in man. F Clin Invest 1987;80:41524 .

17 Lean MEJ, Han TS. Natural sporting ability and predisposition to cardiovascular disorders. $Q \mathcal{F} M$ 1998;91:641-6.

18 Sarna S, Sahi T, Koskenvuo M, et al. Increased life expectancy of world class male athletes. Med Sci Sports Exerc 1993;25:237-44.

19 Saltin B, Gollnick PD. Skeletal muscle adptability, significance for metabolism and performance. In: Peachy LD, Adian RH, Geiger SR, eds. Handbook of physiology: skeletal muscle. Baltimore: Williams and Wilkins, 1983:555-631.

20 World Health Organization. International classification of diseases, eighth revision (ICD-8). Geneva: World Health Organization, 1969

21 World Health Organization. International classification of diseases, ninth revision (ICD-9). Geneva: World Health Organization, 1977

22 Heliövaara M, Reunanen A, Aromaa A, et al. Validity of hospital discharge data in a prospective epidemiological study on stroke and myocardial infarction. Acta Med Scand 1984;216:309-15.

23 Pietilä K, Tenkanen L, Mänttäri M, et al. How to define coronary heart disease in register-based follow-up studies: experience from the Helsinki heart study. Ann Med 1997;29:253-9.

24 Kujala UM, Sarna S, Kaprio J, et al. Heart attacks and lower-limb function in master endurance athletes. Med $S_{c i}$ Sports Exerc 1999;31:1041-6.

25 Finland Central Statistical Office. Alphabetical list of occupations and classification of social class. Helsinki: Finland Central Statistical Office, 1972

26 Kaprio J, Koskenvuo M. A prospective study of psychological and socioeconomic characteristics, health behavior and morbidity in cigarette smokers prior to quitting compared to persistent smokers and non-smokers. 7 Clin Epidemiol $1988 ; 41: 139-50$

27 Sarna S, Kaprio J. Life expectancy of former elite athletes. Sports Med 1994;17:149-51.

28 Rogers MA, Evans WJ. Changes in skeletal muscle with aging: effects of exercise training. Exerc Sport Sci Rev 1993; 2:65-102.

29 Morris JN, Heady JA, Raffle PAB, et al. Coronary heart disease and physical activity of work. Lancet 1953;ii:1053-7, 1111-20.

\section{Take home message}

A previous aptitude for endurance athletic events, unlike the aptitude for power speed events, was associated with protection against coronary heart disease. Evidence is provided that genetic variability may make it easier for some people to achieve a physically active lifestyle and high levels of fitness, as well as favour them with reduced occurrence of coronary heart disease. 